\title{
Faster: Accelerating the Transition from Materials Discovery to Commercial Deployment
}

\section{A TMS Member Perspective}

\section{Alexander H. King}

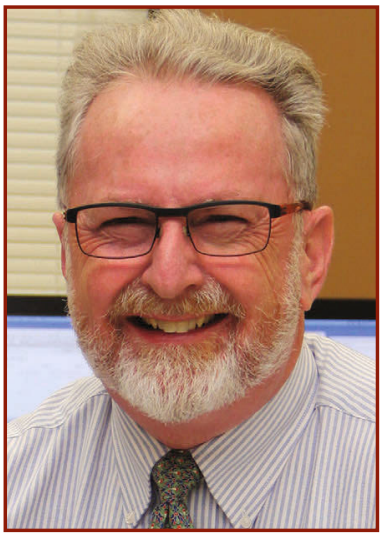

Alex King

\section{Do You Have a Perspective to Share?}

JOM: The Magazine

is seeking case

studies, member

perspectives, and

non-technical

project overviews

with strong industrial

applications. To

suggest an article

idea, contact

Kaitlin Calva,

JOM Magazine

Managing Editor, at

kcalva@tms.org.

\section{The Challenge}

According to popular wisdom, it takes 20 years or more to commercialize a material after its discovery in the lab, and this is used to justify all sorts of research efforts to speed up the process.

The 20-year rule was probably first introduced by Tom Eagar, in a paper in the Massachusetts Institute of Technology's Technology Review ${ }^{1}$ that cites nine examples of 20-year gaps between discovery and commercialization, over a time period from the mid-nineteenth to the late twentieth century. Others have added to the list.

\section{The Response}

It would be easy to conclude that 20 years is the norm, but in fact, it is not. It is not even clear that it is the mode. The examples selected by Eagar clearly identify an opportunity for improvement, but if it were possible to conduct a study of all materials that have been developed in the lab we would certainly find many examples of materials that have taken longer than 20 years to achieve commercial success (if they ever do) and not just a few that have taken significantly less time. Recent efforts by the National Institute of Standards and Technology (NIST) to set standards for measuring the time between discovery and commercialization provide a basis for making the measurements, ${ }^{2}$ but my thesis is that we should spend more effort assessing why some materials go from the lab to the production line much faster, than finding and bemoaning all the cases that take 20 years or more.
In the course of leading the Critical Materials Institute (CMI) for its first five years, I observed at close hand the development and commercialization of a handful of "fast-track materials." I also studied a few other cases that exemplify commercial success in considerably less time than two decades. These all occurred in relatively recent years, but I do not have sufficient data to determine if this represents a trend toward shorter commercialization times. Nevertheless, if we understand these cases and recognize their attributes when they occur elsewhere, perhaps we can take advantage of them and turn fast-tracking into a trend.

\section{Learning from Success}

Each of the following materials, along with several more, are worthy of detailed case studies. They all have different attributes and paths to commercial success, but they also illustrate a few key features and all share one major distinction from the 20 -year cases cited by Eagar. These materials were developed to meet specific needs, rather than being developed because their properties or performance were expected to find revolutionary applications.

- A solder alloy of tin, silver, and copper was invented in $1994^{3}$ and adopted as the worldwide standard for electronics in 2006-twelve years.

- A series of aluminum casting alloys based on the Al-Ce eutectic ${ }^{4}$ was conceived in 2014 and achieved its first commercial sales in 2017-three years.

- Permanent magnets based on the $\mathrm{Nd}_{2} \mathrm{Fe}_{14} \mathrm{~B}$ composition were 
discovered in $1984^{5,6}$ and went into commercial production in 1986- two years.

- In 2014, Apple introduced the iPhone 6 with an aluminum alloy body that could be bent with bare hands. In 2015 it introduced the iPhone 6S with a newly developed and patented 7000-series alloy that was much stiffer. We do not know when the development of the new alloy began, but we can assume a development-tocommercialization time on the order of one year.

The tin, silver, and copper alloy solder was invented in response to environmental pressure to eliminate lead and its use was mandated by regulations introduced in both the European Union and in Japan in 1997- the year that the alloy was patented. The Al-Ce-X alloys were invented to provide highly castable aluminum without the need for distortion-inducing postsolidification heat treatment. $\mathrm{Nd}_{2} \mathrm{Fe}_{14} \mathrm{~B}$ was invented because the production of samarium-cobalt magnets was challenged by a cobalt shortage in 1978. And Apple's stiff aluminum alloy was invented to meet a specific commercial need. All of the materials considered by Eagar provided great new capabilities but there were no products that immediately needed them: their use depended on the development and commercialization of new products and devices that eventually took advantage of the new materials' properties.

Some fast-track materials are only used in the application for which they were initially developed, but some, notably the neodymium magnet composition, have achieved much broader success as their new properties have come to be appreciated, modifications have been developed and new uses have emerged. Early adoption in a single application certainly helps in this process, and this is one of the keys to fast-track commercialization of a new material.

\section{Key Lessons}

Rapid adoption of a material in any specific application depends on several factors. It helps considerably if the material can be used directly in the existing manufacturing process - if it is a "plug-in substitute" for an existing material. Process-compatible substitutes are rare, however, but the smaller the number of process changes that are required, the more easily a new material is adopted. The lead-free solder invented by Miller, Anderson, and Smith melts a few degrees hotter than the lead-tin solder that it replaced, and the higher re-flow temperatures called for other adjustments in the production of integrated circuits. Those process changes were within reach and could be adopted in the designs of next-generation devices, the new solder was quickly adopted.

In a nearly ideal plug-in case, a redemitting phosphor material has been developed as an alternative to europiumbased red phosphors for fluorescent lamps. ${ }^{7}$ This is a product where declining demand and the absence of product updates makes almost any change to the manufacturing process prohibitive, so a plug-in substitute is imperative. Tolerance of the need for process adjustments ultimately depends on the manufacturer's ability to invest in adopting a new material. Adoption is easier in growing markets with frequent product redesigns where new processes are always under development, and harder in stable or shrinking markets with unchanging products. The flexibility of dynamic markets adds another challenge, however. If a material is being developed to target a product with a short redesign cycle like a smart-phone, then that material will have to meet some tough deadlines.

New materials need applications. Without an application, there is no opportunity for commercialization, so we need to assess the opportunities for new materials according to their properties and their potential uses to see which ones might break through the "Eagar barrier." I have tried to do this in a generic form in Table 1, based on the cases described previously.

Research and development efforts aimed at developing new materials are streamlined when they are focused on a single end-use, especially if a manufacturer is involved from the beginning of the process. Developing a new material is, at least at the outset, a process of elimination; and the input from the manufacturer can eliminate candidate materials very quickly,
"New materials need

new applications.

Without an

application, there is

no opportunity for

commercialization..." 
Figure 1. Materials design is a process of down-selection that reduces the burden of materials development efforts. A good strategy is to apply all filters as early as possible in the process. This summarizes the experience of $\mathrm{CMI}$ in developing phosphors for efficient lighting: without input from an industrial partner, synthesis and testing of 12 material systems would have been undertaken. A short

review by the manufacturer cuts this to just three, with the other nine being eliminated for a range of technical and business reasons, reducing the projected experimental work by $75 \%$ and accelerating the R\&D effort by a factor of four.

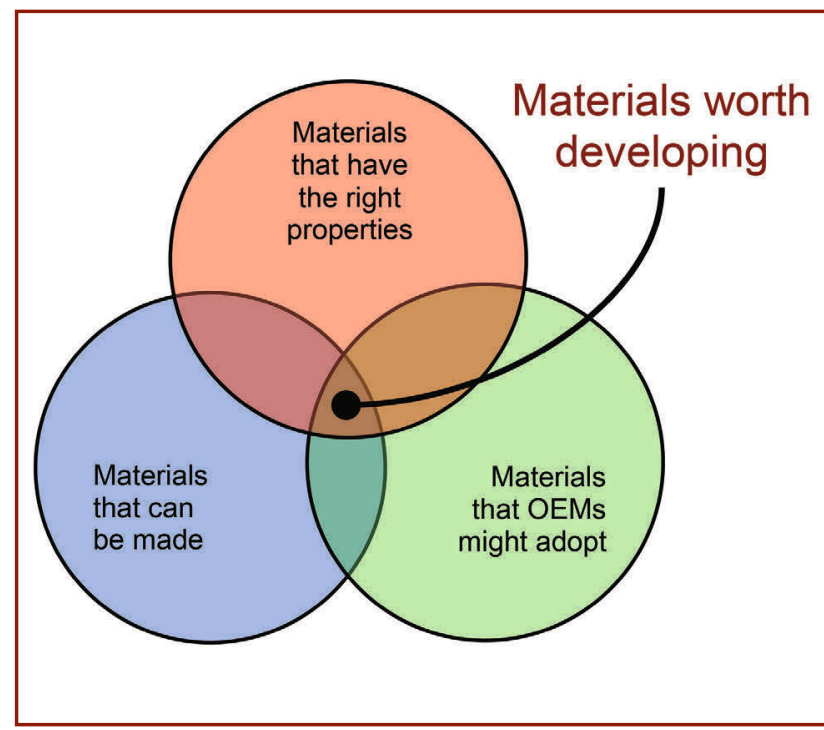

curtailing the need to synthesize and test a large palette of contenders, as illustrated as a Venn diagram in Figure 1. The process of down-selection - the systematic rejection of unacceptable solutions - is achieved by applying filters to the pool of candidates. The sooner we make a No-Go decision, the more the resources that can be applied to the remaining candidates and the early application of available filters accelerates the process.

In one view of the commercialization process, materials are first developed in the lab and then offered to the commercial sector. Progress along the path is characterized by the Technology Readiness Level (TRL) ${ }^{2}$ with research work at low TRL values traditionally being thought of as the domain of research labs, increasingly in universities and national labs, and development work at high-TRL values being the domain of the commercial sector. The standard view of the transition from the lab to the factory is illustrated schematically in Figure 2(a), but many of the materials that have made the transition on the fast track have taken a path more like Figure 2(b), in which there is early involvement from industry, setting the goals and limiting the scope of the low-TRL efforts. At the "back end" of the process, there is also substantial input from the research lab, overcoming barriers to success on the production line, based on detailed understanding of the relationships between structure, properties, and processing of the material. Industry involvement at low TRLs, and researcher 


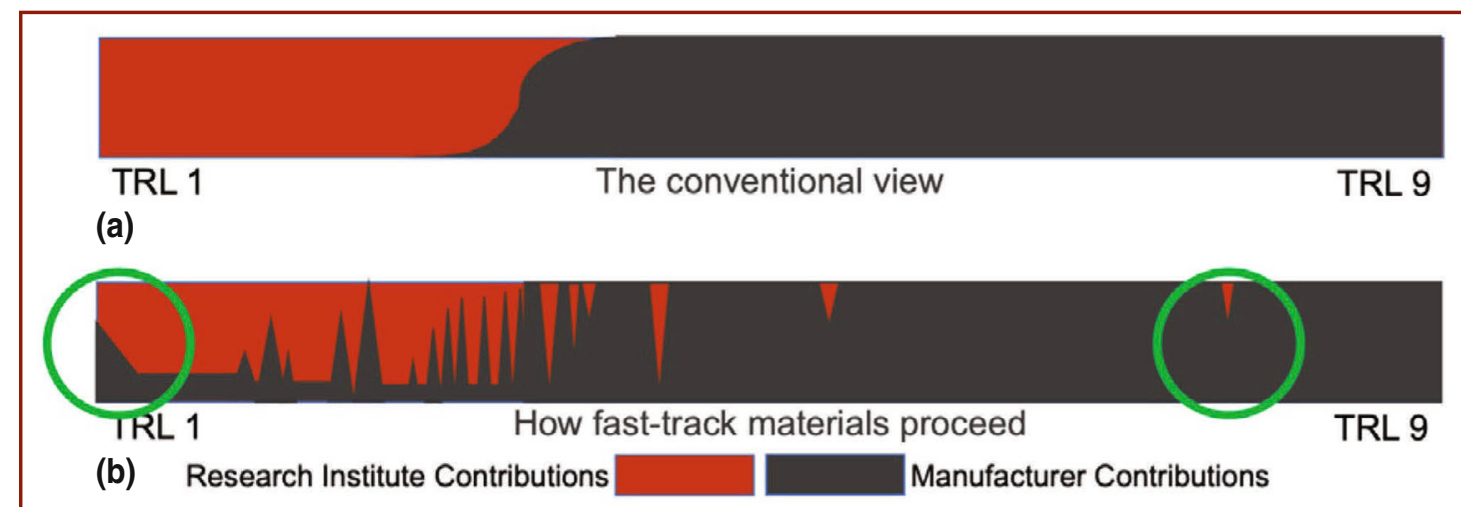

input at higher TRLs are common features of materials whose commercialization succeeds on the fast track.

As seen in Table 1, there are some sweet spots and not-so-sweet spots for the rapid commercialization of new materials, and the sweet spots largely relate to meeting existing manufacturing needs. This is not to cast shade on the value of research aimed at discovering or developing entirely novel materials like high-temperature superconductors, fullerenes, quasicrystals, conducting polymers, ductile ceramics, transparent aluminum, or other Nobelworthy discoveries: these have great potential in the long term, but it takes a long time to develop the applications in which they will have commercial success.

Among the cases of meeting existing needs, however, there are some hints of what might be done to accelerate the commercialization of the truly revolutionary materials: mostly, working closely with end-users as early as possible. What does not work is to invent something and metaphorically throw it over the lab wall in the form of a publication or a patent, expecting investors and manufacturers to find it. The far side of that wall is where the valley of death begins.

\section{What Next?}

The commercialization of new materials can be accelerated if we study the cases where it happens quickly, as opposed to simply cataloging the cases where it does not. I have drawn lessons from a small number of fast-track materials here, but there is plenty of scope to expand on this. There are certainly more lessons in other cases, so I would welcome suggestions about materials that have made it from the lab to the production line in less than
Eagar's canonical 20 years. If you have an example to share, ideally including the discovery and commercialization dates, earliest known research publications, patents, and the names of the commercializing entities, please feel free to contact me at alexking@iastate.edu.

\section{References}

1. T.W. Eagar, Technology Review, 98, 43 (1995).

2. Nexight Group, Quantitative Benchmark for Time to Market (QBTM) for New Materials Innovation: An Analytical Framework. 2016, National Institute of Standards and Technology, https://www.nist.gov/document/ qbtmfinalanalyticalframework011216pdf 3. C.M. Miller, I.E. Anderson, and J.F. Smith, Journal of Electronic Materials, 23, 595 (1994). 4. Z.C. Sims, O.R. Rios, D. Weiss, P.E.A. Turchi, A. Perron, J.R.I. Lee, T.T. Li, J.A. Hammons, M. Bagge-Hansen, T.M. Willey, K. An, Y. Chen, A.H. King, and S.K. Mccall, Materials Horizons, 4, 1070 (2017).

5. J.J. Croat, J.F. Herbst, R.W. Lee, and F.E. Pinkerton, Journal of Applied Physics, 55, 2078 (1984).

6. H. Onodera, Y. Yamaguchi, H. Yamamoto, M. Sagawa, Y. Matsuura, and H. Yamamoto, Journal of Magnetism and Magnetic Materials, 46, 151 (1984).

7. N.J. Cherepy, S.A. Payne, N.M. Harvey, D. Aberg, Z.M. Seeley, K.S. Holliday, I.C. Tran, F. Zhou, H.P. Martinez, J.M. Demeyer, A.D. Drobshoff, A.M. Srivastava, S.J. Camardello, H.A. Comanzo, D.L. Schlagel, and T.A. Lograsso, Optical Materials, 54, 14 (2016).

Alex King is a professor of materials science and engineering at lowa State University. He was the founding director of the Critical Materials Institute, a U.S. Department of Energy (DOE) Energy Innovation Hub headquartered at Ames Laboratory.
Figure 2(a) and (b) Schematic views of how research institutes and manufacturers contribute to the development of new materials across the spectrum to technology readiness levels. Top: the conventional view, which may have a more or less sharp transition. Bottom: the profile seen in fast-track materials. 